\title{
Indiana University Melvin and Bren Simon Cancer Center
}

National Cancer Institute

\section{Source}

National Cancer Institute. Indiana University Melvin and Bren Simon Cancer Center. NCI

Thesaurus. Code C39427.

The Indiana University Cancer Center's mission is to advance the understanding, prevention, and treatment of cancer with patient-centered care, acceleration of promising science, and collaborative educational programs. It became an $\mathrm{NCl}$ designated cancer center in 1999. 